\title{
Staticity Theorem for Non-Rotating Black Holes with Non-Minimally Coupled Self-Interacting Scalar Fields
}

\author{
Eloy Ayón-Beato \\ Departamento de Física \\ Centro de Investigación y de Estudios Avanzados del IPN \\ Apdo. Postal 14-740, C.P. 07000, México, D.F., MEXICO.
}

September 29, 2018

\begin{abstract}
Self-interacting scalar field configurations which are non-minimally coupled $(\zeta \neq 0)$ to the gravity of a strictly stationary black hole with non-rotating horizon are studied. It is concluded that for analytical configurations the corresponding domain of outer communications is static.
\end{abstract}

\section{Introduction}

All the available "no-hair" theorems for non-rotating stationary black holes are based in a staticity hypothesis (see [1, 2, 3, 4, 5] for recent revisions on the subject), i.e., that the asymptotically timelike Killing field $\boldsymbol{k}$, coinciding at the horizon $\mathcal{H}^{+}$with its null generator, must be hypersurface orthogonal in all the domain of outer communications $\langle\langle\mathcal{J}\rangle\rangle$. In this way, in order to establish the uniqueness of the final state of the gravitational collapse of all these systems it is needed to make use of staticity theorems.

Generalizing a previous result by Lichnerowicz for space-times without horizons [6], the staticity theorem corresponding to vacuum black holes was proved by Hawking [7, \&], assuming the existence of strict stationarity, i.e., that the Killing field $\boldsymbol{k}$ is not only timelike $(V \equiv-(\boldsymbol{k} \mid \boldsymbol{k}) \geq 0)$ at infinity, but in all the domain of outer communications $\langle\langle\mathcal{J}\rangle\rangle$.

The extension of this proof to the case of electrovac black holes was proposed by Carter [9], and holds only assuming a condition more restrictive than that one of strict stationarity. This condition occurs to be unphysical, since it is violated even for the black holes of the Reissner-Nordström family when they have electric charges in the interval $4 M / 5<Q<M$. 
Using a Hamiltonian approach, both staticity theorems, for the vacuum and electrovac black holes, have been proved by Sudarsky and Wald 10, 11, 12] without the previous restrictive hypotheses and using only a maximal slice, the existence of which was proved later by Chruściel and Wald [13]. More recently, this last result has been extended, using the same technics, to the axi-dilaton gravity coupled to electromagnetism which is derived from string theory to low energies [14].

For minimally coupled scalar models, staticity theorems has been also proved by Heusler under the strict stationarity hypothesis 15, 16. In this paper we will extend the results by Heusler to the case of non-minimal coupling, assuming again strict stationarity and also analyticity of the scalar fields, establishing in this way that a non-rotating strictly stationary black hole, corresponding to a self-interacting non-minimally coupled scalar field, is static.

For minimally coupled scalar fields there is no need to impose the existence of analyticity for static configurations, since the elliptical nature of the corresponding Einstein-Scalar system guarantees that all the fields are analytical in appropriate coordinates (see the remarks of [17] about the non-vacuum case). The situation is rather different for non-minimally coupled systems, in this case the relevant equations are not necessarily elliptic, and consequently the existence of analyticity must be imposed as a supplementary assumption (see the related discussion in 18]). However, the analyticity hypothesis cannot be considered a too strong one in the study of stationary black holes, since the classification of them rests implicitly in this condition through the Hawking strong rigidity theorem [7, 81. This theorem establishes that the event horizon of a stationary black hole is a Killing horizon, i.e., there exist a Killing field coinciding at the event horizon with the null generators of it (for non-rotating horizons it is the same that the stationary Killing field $\boldsymbol{k}$, but for rotating ones it is different). In order to prove this celebrated theorem, Hawking recurred to the existence of analyticity on all the fields constituting the stationary black hole configuration.

Recent attempts to replace this condition by the more natural one of smoothness have given only positive results for the region interior to the event horizon [19], hence they are of little use in the subject of classification of stationary black-hole exteriors.

The result we will establish is of great utility to eliminate the staticity supposition from the "no-hair" theorems which has been proved for non-minimally coupled scalar fields in presence of non-rotating stationary black holes, see e.g. 20] for the conformal case, and [21, 22, 23, 2] for other results recently derived for more general coupling, where it is not only assumed staticity but also spherical symmetry. 


\section{The Staticity Theorem for Non-Minimally Cou- pled Scalar Fields}

Let us consider the action for a self-interacting scalar field non-minimally coupled to gravity

$$
\mathcal{S}=\frac{1}{2} \int d v\left(\frac{1}{\kappa} R-\left(\nabla_{\mu} \Phi \nabla^{\mu} \Phi+U(\Phi)\right)-\zeta R \Phi^{2}\right),
$$

where $\zeta$ is a real parameter (the values $\zeta=0$ and $\zeta=1 / 6$ correspond to minimal and conformal coupling, respectively).

The variations of this action with respect to the metric and the scalar field, respectively, give rise to the Einstein equations

$$
\left(1-\kappa \zeta \Phi^{2}\right) R_{\nu}^{\mu}=\kappa\left(\nabla_{\nu} \Phi \nabla^{\mu} \Phi+\frac{1}{2} \delta_{\nu}^{\mu}\left(U(\Phi)-\zeta \square \Phi^{2}\right)-\zeta \nabla_{\nu} \nabla^{\mu} \Phi^{2}\right),
$$

and the nonlinear Klein-Gordon equations

$$
\square \Phi-\frac{1}{2} \frac{d U(\Phi)}{d \Phi}-\zeta R \Phi=0 .
$$

From this system of equations it must follows the existence of staticity in the case of strictly stationary black holes with a non-rotating horizon. As it was quoted at the beginning, staticity means that the stationary Killing field $\boldsymbol{k}$ is hypersurface orthogonal, which is equivalent, by the Frobenius theorem, to the vanishing of the twist 1 -form

$$
\omega_{\alpha} \equiv \frac{1}{2} \eta_{\alpha \beta \mu \nu} k^{\beta} \nabla^{\mu} k^{\nu},
$$

where $\boldsymbol{\eta}$ is the volume 4 -form.

In order to exhibit the existence of staticity we will find the explicit dependence of $\boldsymbol{\omega}$ in terms of $\Phi$, by solving the following differential equations which must be satisfied by the twist 1 -form $[9]$

$$
\eta^{\mu \nu \alpha \beta} \nabla_{\alpha} \omega_{\beta}=2 k^{[\mu} \mathcal{R}^{\nu]}
$$

where

$$
\mathcal{R}^{\mu} \equiv k^{\nu} R_{\nu}^{\mu}
$$

is the Ricci vector, that can be evaluated from Einstein equations (2). Using the stationarity of the scalar field

$$
£_{\boldsymbol{k}}(\Phi) \equiv k^{\nu} \nabla_{\nu} \Phi=0,
$$

and the identity

$$
k^{\nu} \nabla_{\nu} \nabla^{\mu} \Phi^{2}=-\nabla^{\mu} k^{\nu} \nabla_{\nu} \Phi^{2},
$$


the Ricci vector can be written as

$$
\left(1-\kappa \zeta \Phi^{2}\right) \mathcal{R}^{\mu}=\kappa\left(\frac{1}{2} k^{\mu}\left(U(\Phi)-\zeta \square \Phi^{2}\right)+\zeta \nabla^{\mu} k^{\nu} \nabla_{\nu} \Phi^{2}\right) .
$$

Replacing this expression in (5), using the Killing vector definition

$$
\nabla^{\mu} k^{\nu}=\nabla^{[\mu} k^{\nu]}
$$

it is obtained the following identity

$$
\left(1-\kappa \zeta \Phi^{2}\right) \eta^{\mu \nu \alpha \beta} \nabla_{\alpha} \omega_{\beta}=2 \kappa \zeta k^{[\mu} \nabla^{\nu} k^{\alpha]} \nabla_{\alpha} \Phi^{2},
$$

which, taking into account definition (何), can be rewritten as

$$
\left(1-\kappa \zeta \Phi^{2}\right) \eta^{\mu \nu \alpha \beta} \nabla_{\alpha} \omega_{\beta}=\frac{2}{3} \kappa \zeta \eta^{\mu \nu \alpha \beta} \nabla_{\alpha} \Phi^{2} \omega_{\beta},
$$

or equivalently, in the language of differential forms as follows

$$
\boldsymbol{d} \boldsymbol{\omega}=\boldsymbol{d}\left(\ln \left[\left(1-\kappa \zeta \Phi^{2}\right)^{-2 / 3}\right]\right) \wedge \boldsymbol{\omega},
$$

where obviously the above expression is valid only in the regions where $\Phi^{2} \neq$ $1 / \kappa \zeta$. The expression (9) can be written also as

$$
\boldsymbol{d}\left(\left(1-\kappa \zeta \Phi^{2}\right)^{2 / 3} \boldsymbol{\omega}\right)=0
$$

where it is understood once more that the equality is valid only when $\Phi^{2} \neq 1 / \kappa \zeta$.

We will analyze now the value of the left hand side of (10) in the regions where $\Phi^{2}=1 / \kappa \zeta$, situation which is only possible for positive values of the non-minimal coupling $(\zeta>0)$. We claim that in the analytic case these regions are composed from a countable union of lower dimensional surfaces, hence, by the continuity of the left hand side of $(10)$, the involved 1 -form is also closed in regions where $\Phi^{2}=1 / \kappa \zeta$.

Lets examine the argument in detail. As it was mentioned at the introductory Section, we suppose that the scalar field $\Phi$ and the metric $\boldsymbol{g}$ are analytical in appropriated coordinates. First it must be noticed that $\Phi^{2} \not \equiv 1 / \kappa \zeta$ in the whole of $\langle\langle\mathcal{J}\rangle\rangle$, i.e., that the square of the scalar field does not take the value $1 / \kappa \zeta$ in every point of the domain of outer communications. This is based in that the converse is in contradiction with the fact that the asymptotic value of the effective gravitational constant

$$
G_{\mathrm{eff}} \equiv \frac{G}{\left(1-\kappa \zeta \Phi^{2}\right)},
$$

must be positive and finite due to the know attractive character of gravity at the asymptotic regions [22]. Since $\Phi^{2} \not \equiv 1 / \kappa \zeta$, it can be shown (see Ref. [26]) from 
the analyticity of $\Phi$, that if the inverse images of the real values $\{ \pm 1 / \sqrt{\kappa \zeta}\}$ under the function $\Phi$,

$$
L_{ \pm} \equiv \Phi^{-1}(\{ \pm 1 / \sqrt{\kappa \zeta}\})
$$

are nonempty, they are composed of a countable union of many 1-dimensional, 2 -dimensional and 3-dimensional analytical submanifolds of $\langle\langle\mathcal{J}\rangle\rangle$. At first sight, 0 -dimensional (point-like) submanifolds are also admissible, but in our case they are excluded by the stationarity. For a proof of the quoted results in $\mathbb{R}^{3}$ see e.g. [26], the extension to $\mathbb{R}^{4}$ does not present any problem.

A direct implication of these results is that in principle the equality $(10)$ is valid just in $\langle\langle\mathcal{J}\rangle\rangle \backslash\left(L_{+} \cup L_{-}\right)$, but by the continuity of the left hand side of (10) in the whole of $\langle\langle\mathcal{J}\rangle\rangle$, and in particular through the lower dimensional surfaces that constitute $L_{ \pm}$, the left hand side of (10) vanishes also in $L_{ \pm}$.

Provided that expression (10) is valid in all the domain of outer communications $\langle\langle\mathcal{J}\rangle\rangle$, it follows from the simple connectedness of this region [25, and the well-known Poincaré lemma, the existence of a global potential $U$ in the whole of $\langle\langle\mathcal{J}\rangle\rangle$ such that

$$
\left(1-\kappa \zeta \Phi^{2}\right)^{2 / 3} \boldsymbol{\omega}=\boldsymbol{d} \boldsymbol{U}
$$

The previous potential $U$ is constant in each connected component of the event horizon $\mathcal{H}^{+}$. This follows from the fact that on the one hand $\boldsymbol{\omega}=0$ in $\mathcal{H}^{+}$, since this region is a Killing horizon whose normal vector coincides with $\boldsymbol{k}$, and on the other hand, as it was previously mentioned (see [7] 8]), in order to that $\mathcal{H}^{+}$be a Killing horizon the scalar field must be analytical, especially at the horizon, hence Eq. (11) implies that $U$ is constant in each connected component of the horizon.

The same result is achieved as well for the asymptotic regions, because any stationary black hole with a bifurcate Killing horizon admits a maximal hypersurface asymptotically orthogonal to the stationary Killing field $\boldsymbol{k}$ [13]. Furthermore, it has been shown [24 that a stationary black hole can be globally extended to other enlarged one possessing a bifurcate Killing horizon.

In what follows we will show that besides the fact that the potential $U$ is constant at the horizon and the asymptotic regions, it will be also constant in the whole of $\langle\langle\mathcal{J}\rangle\rangle$. For the case of minimal coupling $(\zeta=0)$ this results implies directly the staticity (11) as it has been previously proved by Heusler [15]. We will extend his proof to the case of non-minimally coupled to gravity scalar fields.

For every function $f$ and 1 -form $\Omega$, the following identity is satisfied (see the appendix in [16] for the details of the remaining calculations)

$$
\boldsymbol{d}^{\dagger}(f \boldsymbol{\Omega})=f \boldsymbol{d}^{\dagger} \boldsymbol{\Omega}-(\boldsymbol{d} \boldsymbol{f} \mid \boldsymbol{\Omega}),
$$

here $\boldsymbol{d}^{\dagger}=* \boldsymbol{d} *$ stands for the co-differential operator. Applying this expression to the function $U$ and the 1 -form $\Omega / V^{2}$, together with (11), the following can be obtained

$$
\boldsymbol{d}^{\dagger}\left(U \frac{\boldsymbol{\omega}}{V^{2}}\right)=-\frac{\left(1-\kappa \zeta \Phi^{2}\right)^{2 / 3}(\boldsymbol{\omega} \mid \boldsymbol{\omega})}{V^{2}},
$$


where the forthcoming identity has been used (the proof of it can be seen in Ref. [16])

$$
\boldsymbol{d}^{\dagger}\left(\frac{\omega}{V^{2}}\right)=0
$$

Following the same procedure used by Heusler in [15, 16] we will integrate (12) over a spacelike hypersurface $\Sigma$, with volume form $\boldsymbol{i}_{\boldsymbol{k}} \boldsymbol{\eta}$. Taking in consideration that for any stationary 1 -form $\boldsymbol{\Omega}\left(£_{k} \boldsymbol{\Omega}=0\right)$

$$
d^{\dagger} \boldsymbol{\Omega} i_{k} \boldsymbol{\eta}=-d *(k \wedge \Omega),
$$

holds [16], the following relation is obtained applying the Stokes theorem to (12),

$$
\int_{\partial \Sigma} U *\left(\boldsymbol{k} \wedge \frac{\boldsymbol{\omega}}{V^{2}}\right)=\int_{\Sigma} \frac{\left(1-\kappa \zeta \Phi^{2}\right)^{2 / 3}(\boldsymbol{\omega} \mid \boldsymbol{\omega})}{V^{2}} \boldsymbol{i}_{\boldsymbol{k}} \boldsymbol{\eta}
$$

Using now the identity 16

$$
2 *\left(\boldsymbol{k} \wedge \frac{\omega}{V^{2}}\right)=\boldsymbol{d}\left(\frac{\boldsymbol{k}}{V}\right),
$$

the expression (13) can be brought to the form

$$
\frac{1}{2} \int_{\partial \Sigma} U \boldsymbol{d}\left(\frac{\boldsymbol{k}}{V}\right)=\int_{\Sigma} \frac{\left(1-\kappa \zeta \Phi^{2}\right)^{2 / 3}(\boldsymbol{\omega} \mid \boldsymbol{\omega})}{V^{2}} \boldsymbol{i}_{\boldsymbol{k}} \boldsymbol{\eta} .
$$

The boundary $\partial \Sigma$ is constituted at its interior by the event horizon $\mathcal{H}^{+} \cap \Sigma$, and at the infinity by the asymptotic regions. Since the potential $U$ is constant over each one of the connected components of these boundaries, it can be pulled out from each one of the corresponding boundary integrals in the left hand side of $(14)$.

The asymptotic regions and the connected components of the horizon are all topological 2-spheres [8, 25], by this reason the left hand side of (14) vanishes; from Stokes theorem, the integral of an exact form over a manifold without boundary is zero. Thereby, it is satisfied that

$$
\int_{\Sigma} \frac{\left(1-\kappa \zeta \Phi^{2}\right)^{2 / 3}(\boldsymbol{\omega} \mid \boldsymbol{\omega})}{V^{2}} \boldsymbol{i}_{\boldsymbol{k}} \boldsymbol{\eta}=0
$$

The integrand in $(15)$ is non-negative, due to the fact that $\boldsymbol{\omega}$ is a spacelike 1 -form since it is orthogonal by definition to the timelike field $\boldsymbol{k}$ (4). Hence, it follows that (15) is satisfied if and only if the integrand vanishes in $\Sigma$, and by stationarity it also vanishes in all the domain of outer communications $\langle\langle\mathcal{J}\rangle\rangle$, consequently

$$
\left(1-\kappa \zeta \Phi^{2}\right)^{2 / 3}(\boldsymbol{\omega} \mid \boldsymbol{\omega})=0 .
$$

From the previous conclusion (16), it follows that $\boldsymbol{\omega}=0$ in $\langle\langle\mathcal{J}\rangle\rangle \backslash\left(L_{+} \cup L_{-}\right)$, but by the continuity of $\boldsymbol{\omega}$ in all of $\langle\langle\mathcal{J}\rangle\rangle$, and in particular through the lower dimensional surfaces that constitute the regions $L_{ \pm}, \boldsymbol{\omega}$ vanishes also in $L_{ \pm}$and accordingly in all the domain of outer communications $\langle\langle\mathcal{J}\rangle\rangle$. Hence, the staticity theorem is proved. 


\section{Conclusions}

Finally, it is concluded that for a non-rotating strictly stationary black hole with a self-interacting scalar field non-minimally coupled to gravity, the corresponding domain of outer communications is static if analytic field configurations are considered. As in the minimal case [15] this result remains valid when no horizon is present.

\section{Acknowledgments}

The author thanks Alberto García by its incentive in the study of these topics and by its support in the course of the investigation. This research was partially supported by the CONACyT Grant 32138E. The author also thanks all the encouragement and guide provided by his recently late father: Erasmo Ayón Alayo, Ibae Ibae Ibayen Torun.

\section{References}

[1] Heusler, M., Black Hole Uniqueness Theorems (Cambridge Univ. Press, Cambridge 1996).

[2] Bekenstein, J.D., Proceedings of Second Sakharov Conference in Physics, Moscow, eds. I.M. Dremin, A.M. Semikhatov, 761 (World Scientific, Singapore 1997).

[3] Heusler, M., Living Rev. Rel., 1, 1998-6, (http://www.livingreviews.org/Articles/Volume1/1998-6heusler).

[4] Bekenstein, J.D., "Black Holes: Classical Properties, Thermodynamics and Heuristic Quantization," Proceedings of the 9th Brazilian School of Cosmology and Gravitation, Rio de Janeiro, Brazil, gr-qc/9808028 (1998).

[5] Carter, B., Proceedings of 8th Marcel Grossmann Meeting, Jerusalem, ed. T. Piran (World Scientific, Singapore 1999).

[6] Lichnerowicz, A., Théories Relativistes de la Gravitation et de L'Électromagnétisme (Masson \& Cie, Paris 1955).

[7] Hawking, S.W., Commun. Math. Phys., 25, 152 (1972).

[8] Hawking, S.W., Ellis, G.F., The Large Scale Structure of Space-Time (Cambridge Univ. Press 1973).

[9] Carter, B., in Gravitation in Astrophysics (Cargèse Summer School 1986), eds. B. Carter, J.B. Hartle (Plenum, New York 1987).

[10] Sudarsky, D., Wald, R.M., Phys. Rev., D46, 1453 (1992). 
[11] Sudarsky, D., Wald, R.M., Phys. Rev., D47, R3209 (1993).

[12] Wald, R.M., in Proceedings: Directions In General Relativity, eds. B.L. Hu, M.P. Ryan, Jr., C.V. Vishveshwara, T.A. Jacobson (New York, Cambridge Univ. Press, 1993).

[13] Chruściel, P.T., Wald, R.M., Commun. Math. Phys., 163, 561 (1994).

[14] Rogatko, M., Phys. Rev., D58, 044011 (1998).

[15] Heusler, M., Class. Quant. Grav., 10, 791 (1993).

[16] Heusler, M., Straumann, N., Class. Quant. Grav., 10, 1299 (1993).

[17] Müller zum Hagen, H., Proc. Camb. Phil. Soc., 67, 415 (1970).

[18] Zannias, T., J. Math. Phys., 39, 6651 (1998).

[19] Friedrich, H., Racz, I., Wald, R.M., Commun. Math. Phys., 204, 691 (1999).

[20] Zannias, T., J. Math. Phys., 36, 6970 (1995).

[21] Saa, A., J. Math. Phys., 37, 2346 (1996).

[22] Mayo, A.E., Bekenstein, J.D., Phys. Rev., D54, 5059 (1996).

[23] Saa, A., Phys. Rev., D53, 7377 (1996).

[24] Racz, I., Wald, R.M., Class. Quant. Grav., 13, 539 (1996).

[25] Chruściel, P.T., Wald, R.M., Class. Quant. Grav., 11, L147 (1994).

[26] Müller zum Hagen, H., Robinson, D.C., Seifert, H.J., Gen. Rel. Grav., 4, 53 (1973). 